\title{
INDICADORES DE EFICÁCIA DO JOGO “TENHA MODOS" COMO FACILITADOR DO DESENVOLVIMENTO DE HABILIDADES SOCIAIS EM CRIANÇAS
}

DOI: $10.22289 / 2446-922 X . V 8 N 1 A 2$

\author{
Ana Paula Garcia Dellagiustina ${ }^{1}$ \\ Leo Lynce Valle de Lacerda \\ Camilla Volpato Broering
}

\section{RESUMO}

Habilidades sociais são conjuntos de desempenhos apresentados pelos indivíduos em demandas situacionais-interpessoais, sendo a infância uma fase importante para seu desenvolvimento. O objetivo do presente estudo foi avaliar o jogo Tenha Modos como facilitador do desenvolvimento de habilidades sociais. Tratou-se de pesquisa quantitativa com pareamento de dados do tipo "pré" e "pós teste" em uma amostra de 60 crianças de sete a 12 anos. O instrumento de avaliação utilizado foi o Inventário Multimídia de Habilidades Sociais. As crianças foram divididas em três grupos: grupo A:controle; grupo B: com aplicação da estratégia uma vez e grupo C: com aplicação da estratégia duas vezes em um intervalo de duas semanas. Os resultados mostraram diferenças estatisticamente significativas no que se refere ao indicador de frequência da emissão do comportamento socialmente habilidoso para o grupo que utilizou a estratégia lúdica.

Palavras-chave: habilidades sociais; infância; desenvolvimento infantil.

\section{INDICATORS OF EFFECTIVENESS OF A PLAYFUL STRATEGY AS FACILITATOR OF THE DEVELOPMENT OF SOCIAL SKILLS IN CHILDREN}

\section{ABSTRACT}

Social skills are sets of performances presented by individuals in situational-interpersonal demands, and childhood is an important phase for its development. Based on a sample of 60 children aged 7 to 12 years, the present study aimed to evaluate a playful strategy as a facilitator of social skills development. It is a quantitative research with matching of data of the type "pre and post-test". The children were divided into three groups: group A control group B application of the strategy once and group $\mathrm{C}$ application of the strategy twice in a two-week interval. The evaluation tool used was the multimedia inventory of social skills in

\footnotetext{
${ }^{1}$ Endereço eletrônico de contato: anagarciapsi@hotmail.com

Recebido em 31/10/2021. Aprovado pelo conselho editorial para publicação em 06/01/2022.
}

Rev. Psicol Saúde e Debate. Jan., 2022:8(1): 14-28. 
children. Interventions were statistically significant, about the frequency indicator of the emission of socially skillful behavior.

Keywords: social skills; childhood; childhood development.

\section{INDICADORES DE EFICACIA DE UNA ESTRATEGIA LUDICA COMO FACILITADORA DEL DESARROLLO DE HABILIDADES SOCIALES EN NIÑOS}

\section{RESUMEN}

Las habilidades sociales son conjuntos de actuaciones presentados por individuos en demandas situacionales-interpersonales, y la niñez es una fase importante para su desarrollo. Con base en una muestra de 60 niños de 7 a 12 años, el presente estudio tuvo el objetivo de evaluar una estrategia lúdica como facilitadora del desarrollo de habilidades sociales. Se trata de una investigación cuantitativa con paramiento de datos del tipo "pre y post test". Los niños fueron divididos en tres grupos: grupo A control, grupo B aplicación de la estrategia una vez y grupo $\mathrm{C}$ aplicación de la estrategia dos veces en un intervalo de dos semanas. El instrumento de evaluación utilizado fue el inventario multimedia de habilidades sociales en niños. Las77 intervenciones fueron estadísticamente significantes, en lo que se refiere al indicador de frecuencia de la emisión del comportamiento socialmente habilidoso.

Palabras clave: habilidades sociales; infancia; desarrollo infantil.

\section{INTRODUÇÃO}

O termo "habilidades sociais" tem sido utilizado para nominar capacidades comportamentais aprendidas que envolvem interações sociais. Para Del Prette e Del Prette (2018) habilidades sociais são um conjunto de comportamentos sociais aceitos pela respectiva cultura, que proporciona um desempenho social competente e gera resultados positivos para o indivíduo em sua relação com o outro, o que pode vir também a colaborar para a competência social. O desenvolvimento de habilidades sociais pode ocorrer em todas as fases do desenvolvimento humano, considerando que o indivíduo está em constante mudança de comportamento. Dentre estas fases, a infância é considerada a mais importante no desenvolvimento humano (Del Prette \& Del Prette, 2009; Caballo, 2010) devido ao processo de estruturação de comportamentos pró ou antissociais da criança. Tais 
comportamentos irão influenciar o nível de relações interpessoais e comportamentos antissociais apresentados no futuro (Del Prette \& Del Prette, 2008).

Imprescindível falar sobre desempenho social, o qual segundo Del Prette e Del Prette (2017), remete à expressão de comportamentos em situações sociais, desempenhos estes favoráveis ou não para o relacionamento. Já a competência social está relacionada aos efeitos que o desempenho social produz, num sentido avaliativo, o resultado que este gera, se atende às demandas do contexto.

Del Prette e Del Prette (2018) listaram classes e subclasses das principais habilidades sociais: a) de comunicação - fazer e responder perguntas, dar e pedir feedback, gratificar, elogiar, iniciar e manter conversações; b) de civilidade - falar por favor, cumprimentar, despedir-se; c) assertivas - defender os próprios direitos e os dos outros, manejar críticas, discordar, aceitar e recusar pedidos, desculpar-se, admitir falhas e lidar com a raiva do outro; d) empáticas - colocar-se no lugar do outro, expressar compreensão, escutar, manter o contato visual; e) fazer e manter amizade - expressar sentimentos, manter contato e responder ao contato, incentivar confidências, iniciar conversa, realizar e aceitar convites; f) expressar afeto e intimidade - demonstrar afeto por meio do toque, sorriso, demonstrar gentilezas, prezar pelo bem-estar do outro, alimentar o bom humor, lidar com relações íntimas e estabelecer limites quando necessário; g) manejar conflitos e resolver problemas interpessoais - manter a calma, exercitar o autocontrole, identificar e definir o problema, reconhecer em você e no outro comportamentos que levam a ter problemas, procurar possíveis soluções; h) expressar solidariedade - reconhecer as necessidades do outro, participar de campanhas solidárias, oferecer ajuda, apoiar, doar alimentos ou objetos, expressar compaixão; i) coordenar grupos - organizar e dividir tarefas, dar feedback, parafrasear, resumir, saber explicar, incentivar a todos, cobrar desempenho; j) falar em público - usar um tom de voz audível, distribuir o olhar pelos ouvintes, fazer e responder perguntas, utilizar instrumentos audiovisuais adequados. Devido à variabilidade e complexidade das relações interpessoais, sobreposições dos conjuntos são inevitáveis.

Sobre a infância, estudos apontam para déficits no desempenho escolar, disfunções emocionais, comportamento delinquente e uso de drogas (Del Prette \& Del Prette, 2001). Várias técnicas podem ser empregadas para o aprendizado e o aprimoramento de habilidades sociais. Destas Caballo (2007) destaca o treinamento de habilidades sociais (THS) como uma das técnicas de terapia cognitivo-comportamental mais utilizadas, apesar 
de sua complexidade de execução, exigindo conhecimentos de diversas áreas da Psicologia. Dentre os diversos procedimentos do THS, os mais utilizados são: o ensaio comportamental, a modelação e os processos cognitivos. A técnica de treinamento de habilidades sociais é a mais utilizada para o desenvolvimento das habilidades sociais tanto para adultos quanto para crianças.

Batista e Marturano (2015) realizaram um estudo com o objetivo de verificar o potencial de um programa para expandir habilidades sociais e reduzir problemas de comportamento em crianças que são frequentadoras regulares de atividades educativas no contraturno da escola, utilizando histórias infantis e desenhos animados educativos. A propósito dos principais resultados da pesquisa, ou seja, aqueles que apontam para ausência de mudanças significativas nas HS e nos problemas de comportamento das crianças, depois da intervenção, é preciso reconhecer inicialmente a menor probabilidade de sucesso de estratégias focadas exclusivamente na criança, quando comparadas a intervenções sistêmicas. Como os pais são os maiores modelos para os filhos, devem utilizar suas próprias habilidades para ensinar as crianças a se expressarem, apontando, assertivamente, os comportamentos desejáveis e malquistos dos filhos, elogiando quando necessário e solicitando mudanças, explicando suas motivações e as possíveis consequências positivas ou negativas. E certamente, sua opinião sobre as habilidades dos filhos tem grande valia.

Em estudo de LessaHorta, Schäfer, Coelho, Rodrigues, Oliveira e Teixeira (2016) podese verificar as influências que as habilidades sociais ou a falta delas podem causar, pois verificou-se que as duas primeiras condições, luto por morte de filho e sintomas depressivos, estão potencialmente relacionadas uma com a outra e ambas são, frequentemente, determinantes de embotamento e retraimento social significativos. Esses sintomas são caracterizados por inibição comportamental, que contribui consideravelmente para a deterioração do funcionamento social.

Em estudo de Silva e Cavalcante (2017), considerou-se que escolares do sexo masculino, mais jovens, com dificuldades de aprendizagem e problemas de comportamento, tendem apresentar menores médias de habilidades sociais em relação aos do sexo feminino, com mais idade, sem dificuldades de aprendizagem e problemas de comportamento. Neste estudo, as autoras confirmaram, no que se refere ao aspecto escolar, resultados da literatura no que diz respeito ao repertório de habilidades sociais Rev. Psicol Saúde e Debate. Jan., 2022:8(1): 14-28. 
inadequado em crianças que frequentam séries iniciais, neste caso, crianças do segundo ano e que possuem idades entre 6 a 8 anos. Estas crianças estão numa fase de desenvolvimento em que, frequentemente, ainda necessitam de outras pessoas adultas para haver o apaziguamento dos conflitos com pares ou que apresentam estratégias de resolução de conflitos ineficientes, sendo passivas ou agressivas. Além disso, em estudo realizado por Longhini, Rios, Peron e Neufeld (2017), com adolescentes, verificou-se que a vulnerabilidade social e baixo nível socioeconômico, poderia minimizar a influência de outras variáveis, alertando-se para um déficit de comportamentos habilidosos na amostra como um todo, as quais estão na dependência de diversos fatores.

Limberger e Andretta (2017) realizaram um estudo no tema e verificaram que as participantes relataram dificuldades em suas habilidades sociais desde a infância, devido a suas características pessoais e carência de contextos que permitissem o desenvolvimento de um repertório socialmente habilidoso. Com isso, intervenções preventivas deveriam dar ênfase aos momentos críticos do desenvolvimento, a fim de evitar comportamentos de risco. Nessa perspectiva, na avaliação das habilidades sociais, torna-se necessário considerar as comorbidades psiquiátricas, pois estas também podem estar dificultando o desempenho de tais habilidades (Limberger, 2016).

Por sua vez, as habilidades sociais de crianças em idade escolar como forma de contribuir para a prevenção de problemas de comportamento e, consequentemente, para o desenvolvimento socioemocional infantil correspondem aos achados que instigam 0 desenvolvimento de programas de intervenções que podem trazer ganhos tanto para a própria criança quanto para o âmbito escolar e familiar (Casali-Robalinho, Del Prette, \& Del Prette, 2015).

Todos estes estudos em conjunto, levam a pensar a importância de se existir recursos que contemplem e viabilizem o desenvolvimento de habilidades sociais na mais tenra idade, como forma de prevenir possíveis riscos ao desenvolvimento e futuros problemas, como os verificados neste artigo. Dellagiustina e Broering (2017) elaboraram um jogo intitulado "Tenha Modos" considerando a importância das habilidades sociais no desenvolvimento infantil, tanto no contexto familiar quanto escolar. As tarefas do jogo proporcionam o treino de habilidades sociais em crianças, principalmente comportamento educado, assertivo, colaborador e empático nas relações verticais (com pais, avós, professores) e horizontais (com pares). O cumprimento das tarefas produzem recompensas (brinquedos e/ou amigos). 
O campo das habilidades sociais no Brasil vem crescendo nos últimos dez anos. Estudos de revisão como os de Murta e Goias (2005) e Bolsoni-Silva et al. (2006) analisaram a produção nacional e apontaram uma produção crescente, com ênfase em pesquisas de delineamento experimental com pré e pós teste, mensurando assim a eficácia dos programas de treinamento em habilidades sociais em níveis de prevenção primários, secundários e terciários. Dentre estes, Gonçalves e Murta (2008) propuseram um modelo de 20 sessões estruturadas com base em vivências de grupo, em que obtiveram resultados positivos frente aos comportamentos pró-sociais das crianças.

Del Prette e Del Prette (2008) estabeleceram um conjunto de classes de habilidades específicas na infância passíveis de serem desenvolvidas, necessárias à obtenção de um desempenho social adequado ao seu ambiente. Este é composto por sete classes de habilidades sociais: autocontrole e expressividade emocional; civilidade; empatia; assertividade; fazer amizades; solução de problemas interpessoais e habilidades sociais acadêmicas.

Frente a escassez de instrumentos de avaliação de habilidades sociais e competência social de crianças para avaliar a eficácia dos programas de treinamento de habilidades sociais, foi criado o sistema multimídia de habilidades sociais em crianças. $\mathrm{O}$ instrumento tem como base conceitual o conjunto de habilidades sociais infantis, desempenho social e competência social. Ao avaliar o instrumento, Del Prette e Del Prette (2012) apontaram satisfatória consistência interna e eficácia na aplicabilidade do instrumento para amostra brasileira. O presente estudo avaliou a eficácia de uma estratégia lúdica respaldada na terapia cognitiva-comportamental como estratégia de aprimoramento e desenvolvimento de habilidades sociais infantis.

\section{MATERIAIS E MÉTODOS}

\subsection{Participantes}

Trata-se de pesquisa quantitativa com pareamento de dados do tipo "pré e pós teste" a partir da aplicação de instrumento padronizado antes e após a aplicação de um jogo terapêutico. Participaram do estudo crianças usuárias dos serviços de uma organização não governamental do sul do país no ano de 2017, 23 crianças do sexo feminino e 37 do 
sexo masculino com idades entre sete a doze anos distribuídas uniformemente entre os grupos de estudo e controle.

\subsection{Instrumentos}

O instrumento de coleta de dados foi o Inventário Multimídia de Habilidades Sociais (IMHSC) adequado a faixa etária de sete a 12 anos (Del Prette \& Del Prette, 2012). Este instrumento foi disponibilizado em versão impressa e digital (CD rom). Possui 21 itens de situações interpessoais, representados de maneira interativa (vídeo) para a criança (autoavaliação) e de maneira impressa para avaliação de pais e professores. Neste estudo foi utilizado a versão digital (autoavaliação).

O instrumento divide-se em quatro subescalas de avaliação: F1-Empatia/Civilidade; F2Assertividade de Enfrentamento; F3-Autocontrole; F4-Participação. Cada subescala apresenta o vídeo da interação social seguido da classificação pela criança em três alternativas comportamentais: habilidosa, não habilidosa ativa e não habilidosa passiva. A criança ainda deve indicar a frequência, adequação e dificuldade da emissão do comportamento apresentado.

\subsection{Procedimento}

\subsubsection{Coleta de dados}

Os dados foram coletados na própria instituição não governamental em sala de atendimento psicológico, com a presença do pesquisador e da criança. Previamente à coleta de dados foram determinados três grupos, cada qual com 20 crianças. A coleta ocorreu em três fases: Na primeira fase, foi aplicado o pré-teste IMHSC em todas as crianças participantes do estudo. Na segunda fase, o grupo A passou pela aplicação da estratégia lúdica uma única vez, o grupo B passou pela estratégia lúdica duas vezes em um intervalo de duas semanas e o grupo C (controle) não teve contato com a estratégia lúdica. A terceira fase e etapa final da coleta foi a realização do pós-teste IMHSC com intervalo de uma semana após a realização da estratégia lúdica para Grupo A e B e para o Grupo C em um intervalo de duas semanas da primeira avaliação. 


\subsubsection{Análise dos dados}

Os dados foram registrados em planilha Excel®, computadas as frequências de crianças que estiveram abaixo, no valor mediano e acima da amostra de referência do IMHSC- (Del Prette, 2012) e observadas as diferenças entre grupos. Posteriormente os dados foram analisados por meio da prova não paramétrica de Wilcoxon na comparação dos escores antes e depois da aplicação do jogo, com o auxílio do aplicativo Statistica versão 12. O nível de significância adotado foi de $5 \%$.

\subsubsection{Considerações Éticas}

Antes da aplicação do instrumento foi realizado linha de base junto as crianças, conversa ética explicativa aos responsáveis, apresentado o Termo de Consentimento Livre e Esclarecido TCLE, bem como o Termo de Assentimento Livre e Esclarecido à criança. $A$ coleta de dados respeitou as diretrizes e normas estabelecidas na Resolução no68415517.8.0000.0120 que regulamenta as pesquisas envolvendo seres humanos. A pesquisa foi aprovada pelo Comitê de Ética em Pesquisa, número do parecer: 2.118.128.

\section{RESULTADOS}

A Tabela 1 apresenta a média e os intervalos de confiança dos escores de habilidades sociais entre os grupos de acordo com as subescalas do indicador de adequação, antes e depois da aplicação da estratégia lúdica. No grupo B houve queda significativa na subescala autocontrole nas alternativas comportamentais habilidosa e não habilidosa passiva, resultado esperado visto que o jogo trabalha com a adequação da emissão do comportamento socialmente hábil caracterizada como somente como certo ou errado, diferenciando-se do tipo de resposta que o instrumento utiliza: certo, errado e mais ou menos.

Tabela 1 - Dados relacionados aos escores de habilidades sociais entre os grupos, antes e depois da aplicação da estratégia lúdica, de acordo com as subescalas do indicador de adequação.

\begin{tabular}{llll}
\hline Reação/Subescala & A & B & Controle \\
\cline { 2 - 4 }
\end{tabular}




\begin{tabular}{|c|c|c|c|c|c|c|}
\hline & Antes & Depois & Antes & Depois & Antes & Depois \\
\hline \multicolumn{7}{|l|}{ Habilidosa } \\
\hline Empatia/Civilidade & $\begin{array}{l}1,66 \\
(1,43-1,88)\end{array}$ & $\begin{array}{l}1,72 \\
(1,51-1,93)\end{array}$ & $\begin{array}{l}1,88 \\
(1,78-1,97)\end{array}$ & $\begin{array}{l}1,85 \\
(1,75-1,95)\end{array}$ & $\begin{array}{l}1,41 \\
(1,05-1,77)\end{array}$ & $\begin{array}{l}1,23 \\
(0,88-1,58)\end{array}$ \\
\hline Assertividade/Enfrentamento & $\begin{array}{l}1,25 \\
(1,07-1,42)\end{array}$ & $\begin{array}{l}1,38 \\
(1,17-1,58)\end{array}$ & $\begin{array}{l}1,21 \\
(1,00-1,42)\end{array}$ & $\begin{array}{l}1,12 \\
(0,89-1,35)\end{array}$ & $\begin{array}{l}1,09 \\
(0,82-1,37)\end{array}$ & $\begin{array}{l}1,09 \\
(0,81-1,37)\end{array}$ \\
\hline Autocontrole & $\begin{array}{l}1,26 \\
(1,06-1,46)\end{array}$ & $\begin{array}{l}1,27 \\
(1,01-1,52)\end{array}$ & $\begin{array}{l}1,38^{a} \\
(1,19-1,56)\end{array}$ & $\begin{array}{l}1,15^{\mathrm{a}} \\
(0,99-1,31)\end{array}$ & $\begin{array}{l}0,98 \\
(0,65-1,31)\end{array}$ & $\begin{array}{l}1,10 \\
(0,80-1,41)\end{array}$ \\
\hline Participação & $\begin{array}{l}1,65 \\
(1,43-1,88)\end{array}$ & $\begin{array}{l}1,73 \\
(1,49-1,96)\end{array}$ & $\begin{array}{l}1,80 \\
(1,64-1,96)\end{array}$ & $\begin{array}{l}1,75 \\
(1,56-1,94)\end{array}$ & $\begin{array}{l}1,42 \\
(1,47-1,78)\end{array}$ & $\begin{array}{l}1,27 \\
(0,87-1,67)\end{array}$ \\
\hline \multicolumn{7}{|l|}{ Não habilidosa passiva } \\
\hline Empatia/Civilidade & $\begin{array}{l}0,60 \\
(0,41-0,79)\end{array}$ & $\begin{array}{l}0,58 \\
(0,36-0,800\end{array}$ & $\begin{array}{l}0,44 \\
(0,28-0,61)\end{array}$ & $\begin{array}{l}0,33 \\
(0,15-0,52)\end{array}$ & $\begin{array}{l}0,43 \\
(0,20-0,66)\end{array}$ & $\begin{array}{l}0,40 \\
(0,16-0,64)\end{array}$ \\
\hline Assertividade/Enfrentamento & $\begin{array}{l}0,53 \\
(0,40-0,66)\end{array}$ & $\begin{array}{l}0,67 \\
(0,22-0,54)\end{array}$ & $\begin{array}{l}0,55 \\
(0,38-0,72)\end{array}$ & $\begin{array}{l}0,38 \\
(0,22-0,54)\end{array}$ & $\begin{array}{l}0,58 \\
(0,39-0,77)\end{array}$ & $\begin{array}{l}0,48 \\
(0,36-0,60)\end{array}$ \\
\hline Autocontrole & $\begin{array}{l}0,88 \\
(0,66-1,10)\end{array}$ & $\begin{array}{l}1,04 \\
(0,82-1,26)\end{array}$ & $\begin{array}{l}1,03^{b} \\
(0,84-1,21)\end{array}$ & $\begin{array}{l}0,76^{\mathrm{b}} \\
(0,59-0,94)\end{array}$ & $\begin{array}{l}0,92 \\
(0,69-1,15)\end{array}$ & $\begin{array}{l}0,80 \\
(0,60-1,00)\end{array}$ \\
\hline Participação & $\begin{array}{l}0,53 \\
(0,31-0,75)\end{array}$ & $\begin{array}{l}0,67 \\
(0,48-0,86)\end{array}$ & $\begin{array}{l}0,60 \\
(0,34-0,86)\end{array}$ & $\begin{array}{l}0,47 \\
(0,30-0,63)\end{array}$ & $\begin{array}{l}0,67 \\
(0,44-0,91)\end{array}$ & $\begin{array}{l}0,61 \\
(0,38-0,84)\end{array}$ \\
\hline \multicolumn{7}{|l|}{ Não habilidosa ativa } \\
\hline Empatia/Civilidade & $\begin{array}{l}0,16 \\
(0,05-0,27)\end{array}$ & $\begin{array}{l}0,12 \\
(0,01-0,23)\end{array}$ & $\begin{array}{l}0,11 \\
(0,00-0,22)\end{array}$ & $\begin{array}{l}0,04 \\
(0,00-0,10)\end{array}$ & $\begin{array}{l}0,24 \\
(0,07-0,41)\end{array}$ & $\begin{array}{l}0,21 \\
(0,04-0,39)\end{array}$ \\
\hline Assertividade/Enfrentamento & $\begin{array}{l}0,40 \\
(0,19-0,62)\end{array}$ & $\begin{array}{l}0,24 \\
(0,10-0,37)\end{array}$ & $\begin{array}{l}0,11 \\
(0,01-0,21)\end{array}$ & $\begin{array}{l}0,21 \\
(0,08-0,34)\end{array}$ & $\begin{array}{l}0,29 \\
(0,07-0,51)\end{array}$ & $\begin{array}{l}0,33 \\
(0,04-0,62)\end{array}$ \\
\hline Autocontrole & $\begin{array}{l}0,30 \\
(0,07-0,52)\end{array}$ & $\begin{array}{l}0,10 \\
(0,03-0,18)\end{array}$ & $\begin{array}{l}0,06 \\
(0,00-0,13)\end{array}$ & $\begin{array}{l}0,10 \\
(0,00-0,20)\end{array}$ & $\begin{array}{l}0,18 \\
(0,10-0,26)\end{array}$ & $\begin{array}{l}0,25 \\
(0,05-0,45)\end{array}$ \\
\hline Participação & $\begin{array}{l}0,51 \\
(0,33-0,70)\end{array}$ & $\begin{array}{l}0,35 \\
(0,17-0,53)\end{array}$ & $\begin{array}{l}0,25 \\
(0,09-0,41)\end{array}$ & $\begin{array}{l}0,23 \\
(0,09-0,38)\end{array}$ & $\begin{array}{l}0,25 \\
(0,04-0,45)\end{array}$ & $\begin{array}{l}0,40 \\
(0,17-0,64)\end{array}$ \\
\hline
\end{tabular}

${ }^{*}$ valores significativos: ${ }^{a} p=0,0189 ;{ }^{b} p=0,0331$. Prova de Wilcoxon, $5 \%$.

Fonte: dados da pesquisa

$\mathrm{Na}$ Tabela 2 são mostrados a média e intervalos de confiança dos escores de habilidades sociais entre os grupos, de acordo com as subescalas do indicador de frequência. Observa-se no grupo B uma tendência ao aumento da frequência habilidosa dos participantes em todas as subescalas, com mudança significativa na subescala empatia/civilidade. No grupo A este aumento foi observado na reação não habilidosa 
passiva nas subescalas assertividade e autocontrole. Ressalta-se que não houve mudança significativa no grupo controle.

Tabela 2 - Dados relacionados aos escores de habilidades sociais entre os grupos, antes e depois da aplicação da estratégia lúdica, de acordo com as subescalas do indicador de frequência

\begin{tabular}{|c|c|c|c|c|c|c|}
\hline \multirow{2}{*}{ Reação/Subescala } & \multicolumn{2}{|l|}{ A } & \multicolumn{2}{|l|}{ B } & \multicolumn{2}{|l|}{ Controle } \\
\hline & Antes & Depois & Antes & Depois & Antes & Depois \\
\hline \multicolumn{7}{|l|}{ Habilidosa } \\
\hline Empatia/Civilidade & $\begin{array}{l}1,61^{\mathrm{a}} \\
(1,44-1,77)\end{array}$ & $\begin{array}{l}1,28^{a} \\
(1,07-1,48)\end{array}$ & $\begin{array}{l}1,35^{\mathrm{b}} \\
(1,13-1,57)\end{array}$ & $\begin{array}{l}1,55^{\mathrm{b}} \\
(1,37-1,73)\end{array}$ & $\begin{array}{l}1,50 \\
(1,21-1,79)\end{array}$ & $\begin{array}{l}1,19 \\
(0,88-1,49)\end{array}$ \\
\hline Assertividade/Enfrentamento & $\begin{array}{l}0,77 \\
(0,55-0,98)\end{array}$ & $\begin{array}{l}0,91 \\
(0,76-1,05)\end{array}$ & $\begin{array}{l}0,68 \\
(0,51-0,85)\end{array}$ & $\begin{array}{l}0,74 \\
(0,54-0,94)\end{array}$ & $\begin{array}{l}0,72 \\
(0,48-0,96)\end{array}$ & $\begin{array}{l}0,67 \\
(0,45-0,89)\end{array}$ \\
\hline Autocontrole & $\begin{array}{l}0,97 \\
(0,78-1,15)\end{array}$ & $\begin{array}{l}1,05 \\
(0,87-1,23)\end{array}$ & $\begin{array}{l}0,80 \\
(0,67-0,93)\end{array}$ & $\begin{array}{l}0,98 \\
(0,77-1,18)\end{array}$ & $\begin{array}{l}0,88 \\
(0,76-1,00)\end{array}$ & $\begin{array}{l}0,80 \\
(0,52-1,08)\end{array}$ \\
\hline Participação & $\begin{array}{l}1,38 \\
(1,12-1,63)\end{array}$ & $\begin{array}{l}1,29 \\
(1,10-1,48)\end{array}$ & $\begin{array}{l}1,20 \\
(0,94-1,46)\end{array}$ & $\begin{array}{l}1,35 \\
(1,11-1,60)\end{array}$ & $\begin{array}{l}1,33 \\
(1,15-1,45)\end{array}$ & $\begin{array}{l}1,07 \\
(0,74-1,41)\end{array}$ \\
\hline \multicolumn{7}{|l|}{ Não habilidosa passiva } \\
\hline Empatia/Civilidade & $\begin{array}{l}0,44 \\
(0,25-0,62)\end{array}$ & $\begin{array}{l}0,42 \\
(0,28-0,56)\end{array}$ & $\begin{array}{l}0,27 \\
(0,11-0,43)\end{array}$ & $\begin{array}{l}0,29 \\
(0,15-0,43)\end{array}$ & $\begin{array}{l}0,45 \\
(0,21-0,69)\end{array}$ & $\begin{array}{l}0,53 \\
(0,23-0,84)\end{array}$ \\
\hline Assertividade/Enfrentamento & $\begin{array}{l}0,41^{c} \\
(0,25-0,56)\end{array}$ & $\begin{array}{l}0,59^{c} \\
(0,43-0,76)\end{array}$ & $\begin{array}{l}0,38 \\
(0,21-0,55)\end{array}$ & $\begin{array}{l}0,32 \\
(0,17-0,47)\end{array}$ & $\begin{array}{l}0,45 \\
(0,26-0,64)\end{array}$ & $\begin{array}{l}0,48 \\
(0,28-0,68)\end{array}$ \\
\hline Autocontrole & $\begin{array}{l}0,50^{d} \\
(0,29-0,71)\end{array}$ & $\begin{array}{l}0,71^{d} \\
(0,50-0,91)\end{array}$ & $\begin{array}{l}0,55 \\
(0,38-0,72)\end{array}$ & $\begin{array}{l}0,63 \\
(0,43-0,82)\end{array}$ & $\begin{array}{l}0,60 \\
(0,43-0,67)\end{array}$ & $\begin{array}{l}0,59 \\
(0,39-0,79)\end{array}$ \\
\hline Participação & $\begin{array}{l}0,36 \\
(0,21-0,51)\end{array}$ & $\begin{array}{l}0,63 \\
(0,48-0,77)\end{array}$ & $\begin{array}{l}0,47 \\
(0,26-0,68)\end{array}$ & $\begin{array}{l}0,30 \\
(0,13-0,47)\end{array}$ & $\begin{array}{l}0,67 \\
(0,37-0,97)\end{array}$ & $\begin{array}{l}0,76 \\
(0,45-1,08)\end{array}$ \\
\hline \multicolumn{7}{|l|}{ Não habilidosa ativa } \\
\hline Empatia/Civilidade & $\begin{array}{l}0,07 \\
(0,02-0,12)\end{array}$ & $\begin{array}{l}0,13 \\
(0,04-0,21)\end{array}$ & $\begin{array}{l}0,06 \\
(0,00-0,13)\end{array}$ & $\begin{array}{l}0,06 \\
(0,01-0,12)\end{array}$ & $\begin{array}{l}0,25 \\
(0,09-0,41)\end{array}$ & $\begin{array}{l}0,36 \\
(0,16-0,55)\end{array}$ \\
\hline Assertividade/Enfrentamento & $\begin{array}{l}0,31 \\
(0,11-0,50)\end{array}$ & $\begin{array}{l}0,34 \\
(0,15-0,53)\end{array}$ & $\begin{array}{l}0,15 \\
(0,01-0,29)\end{array}$ & $\begin{array}{l}0,22 \\
(0,07-0,37)\end{array}$ & $\begin{array}{l}0,40 \\
(0,16-0,65)\end{array}$ & $\begin{array}{l}0,37 \\
(0,15-0,58)\end{array}$ \\
\hline Autocontrole & $\begin{array}{l}0,29 \\
(0,05-0,52)\end{array}$ & $\begin{array}{l}0,25 \\
(0,03-0,48)\end{array}$ & $\begin{array}{l}0,06 \\
(0,00-0,13)\end{array}$ & $\begin{array}{l}0,11 \\
(0,00-0,23)\end{array}$ & $\begin{array}{l}0,35 \\
(0,12-0,59)\end{array}$ & $\begin{array}{l}0,28 \\
(0,04-0,52)\end{array}$ \\
\hline Participação & $\begin{array}{l}0,44 \\
(0,28-0,59)\end{array}$ & $\begin{array}{l}0,32 \\
(0,19-0,45)\end{array}$ & $\begin{array}{l}0,12 \\
(0,01-0,22)\end{array}$ & $\begin{array}{l}0,15 \\
(0,06-0,24)\end{array}$ & $\begin{array}{l}0,32 \\
(0,08-0,57)\end{array}$ & $\begin{array}{l}0,40 \\
(0,14-0,65)\end{array}$ \\
\hline
\end{tabular}

Rev. Psicol Saúde e Debate. Jan., 2022:8(1): 14-28. 
${ }^{*}$ valores significativos: ${ }^{a} p=0,0105 ;{ }^{b} p=0,0279 ;{ }^{c} p=0,0465 ;{ }^{d} p=0,0186$. Prova de Wilcoxon, $5 \%$.

Fonte: dados da pesquisa

Com relação aos escores do indicador de dificuldade (Tabela 3) não houve mudança significativa nos três grupos, apesar do aumento discreto na subescala assertividade e autocontrole no grupo B, resultado correlacionado com o aumento do escore no indicador da adequação (Tabela 1) e queda no indicador da frequência (Tabela 2).

Tabela 3 - Dados relacionados aos escores de habilidades sociais entre os grupos, antes e depois da aplicação da estratégia lúdica, de acordo com as subescalas do indicador de dificuldade

\begin{tabular}{lllllll}
\hline \multirow{2}{*}{ Subescala } & $\mathrm{A}$ & & $\mathrm{B}$ & \multicolumn{3}{c}{ Controle } \\
& Antes & Depois & Antes & Depois & Antes & Depois \\
\hline Empatia/Civilidade & 0,39 & 0,49 & 0,44 & 0,41 & 0,45 & 0,38 \\
& $(0,16-0,61)$ & $(0,24-0,74)$ & $(0,21-0,66)$ & $(0,20-0,61)$ & $(0,30-0,55)$ & $(0,18-0,59)$ \\
Assertividade/Enfrentamento & 0,70 & 0,42 & 0,56 & 0,71 & 0,53 & 0,45 \\
& $(0,44-0,96)$ & $(0,21-0,63)$ & $(0,35-0,77)$ & $(0,41-1,01)$ & $(0,29-0,78)$ & $(0,24-0,67)$ \\
Autocontrole & 0,52 & 0,52 & 0,54 & 0,65 & 0,70 & 0,51 \\
& $(0,31-0,74)$ & $(0,29-0,75)$ & $(0,35-0,72)$ & $(0,39-0,91)$ & $(0,40-1,00)$ & $(0,25-0,78)$ \\
Participação & 0,56 & 0,53 & 0,52 & 0,47 & 0,53 & 0,44 \\
& $(0,28-0,83)$ & $(0,28-0,78)$ & $(0,29-0,75)$ & $(0,23-0,70)$ & $(0,20-0,72)$ & $(0,19-0,69)$ \\
\hline
\end{tabular}

Nota: em todas as comparações as diferenças não foram significativas. Prova de Wilcoxon, $5 \%$.

Fonte: dados da pesquisa

\section{DISCUSSÃO}

Os estudos acerca das habilidades sociais não abordam tempo de duração e nenhuma especificidade acerca de estratégias lúdicas a serem utilizadas nos modelos de programa Rev. Psicol Saúde e Debate. Jan., 2022:8(1): 14-28. 
de Treinamento de Habilidades Sociais (THS). No entanto, Del Prette e Del Prette (2018) afirmam que o THS é um tipo de terapia considerada breve e resultados eficazes podem ser esperados com duas seções de uma hora e meia a duas horas por semana durante quatro a cinco meses, ou um programa de seis a oito meses quando a seção for semanal.

Os resultados indicaram que após a aplicação do jogo ocorreram mudanças no repertório social encontrado. No que se refere ao indicador adequação não foi observado nenhuma mudança positiva. Observou-se queda significativa na subescala autocontrole, possivelmente devido a influência da ausência de uma reação do tipo mais ou menos no jogo como foi apresentada pelo instrumento e desta forma contribuindo para a diminuição da escala, e consequentemente interferindo no escore do indicador.

Pôde-se observar possível influência do jogo no que se refere ao indicador de frequência: houve a diminuição do indicador da reação habilidosa para o grupo controle, e aumento da emissão do comportamento socialmente habilidoso em todas as subescalas do grupo B. Este resultado contrapõe-se com o estudo realizado por Cia et al. (2006) o qual objetivou-se por comparar indicadores de envolvimento de pais com filhos com repertório de habilidades sociais e que sinalizou correlação positiva entre os indicadores de adequação e frequência nas quatro subescalas apresentadas pelo IMHSC.

No estudo realizado por Barreto et al. (2011) no qual investigaram indicadores acerca das habilidades sociais na comorbidade entre dificuldade de aprendizagem e problemas de comportamento em crianças de oito a 12 anos obteve-se uma correlação de déficit entre a subescala empatia e civilidade e assertividade de enfrentamento. No presente estudo todas as subescalas do indicador frequência mostraram mudança positiva, com diferença significativa na subescala de empatia. Del Prette e Del Prette, (2018) afirmaram que as duas subescalas empatia e civilidade e assertividade são integrantes, pois possuem caráter complementar abrangendo as necessidades do indivíduo e do interlocutor. Pode-se associar esta afirmação com o formato da estratégia lúdica, na qual, para sua prática, é necessário exercer a empatia através da representação de situações sociais em que a criança necessita colocar-se no lugar do outro.

O indicador de dificuldade não obteve variação significativa em nenhum dos grupos. Neste sentido o estudo demonstrou a influência do jogo ao trabalhar a frequência da emissão do comportamento socialmente habilidoso. 
Foram consideradas as principais limitações deste estudo: o número amostral, e em consequência deste, o tipo de prova não paramétrica utilizada na análise dos dados, cujo poder de teste pode não ter sido suficiente para detectar diferenças entre os grupos; a influência do gênero e idade dos participantes com relação às habilidades sociais, mesmo considerando a homogeneização dos grupos, o que pode ter aumentado a variabilidade interna dos grupos e com isso minimizado possíveis diferenças entre estes e por fim a aplicação do IMHSC e da estratégia lúdica pela mesma pessoa, o que pode ter acarretado efeito de desabilidade social nos participantes. Por fim, o escasso número de trabalhos que empregaram estratégias lúdicas para o desenvolvimento de habilidades sociais dificultou análises comparativas.

\section{CONSIDERAÇÕES FINAIS}

Concluiu-se que dos indicadores de adequação, frequência e dificuldade, foram encontradas mudanças positivas em quatro subescalas para adequação e seis subescalas para frequência, com variação significativa somente no indicador de frequência: civilidade/empatia na reação habilidosa e assertividade e autocontrole na reação não habilidosa passiva. Tal resultado configurou uma mudança no repertório social dos sujeitos, notadamente na frequência da emissão do comportamento. Estratégias como a que foi aqui investigada podem se tornar recursos práticos e interessantes, na medida que favorecem a prática psicológica nesta faixa etária e possibilitam o desenvolvimento de habilidades por meio de uma estratégia lúdica aplicável por pais e responsáveis no ambiente familiar.

\section{REFERÊNCIAS}

Barreto, S. O., Freitas, L. C., Del Prette, Z. A. P. (2011). Habilidades sociais na comorbidade entre dificuldades de aprendizagem e problemas de comportamento: uma avaliação multimodal. [Social skills in the comorbidity between learning disabilities and behavior problems: a multimodal assessment] Psico, 42 (4), 503-510.

Batista, S. V., Marturano, E. M. (2015). Intervenção para promover habilidades sociais e reduzir problemas de comportamento de crianças em um núcleo social. Pesquisas e Práticas Psicossociais, 10(2), 313-326.

Bolsoni-Silva, A. T., Del Prette, Z. A. P., Del Prette, G., Montagner, A. R., Bandeira, M., Del Prette, A. (2006). Habilidades sociais no Brasil: Uma análise dos estudos publicados 
em periódicos. In M. Bandeira., Z. A. P. Del Prette., A. Del Prette, (Orgs.), Estudos sobre habilidades sociais e relacionamento interpessoal. [Studies on social skills and interpersonal relationships] (1th ed. pp. 1-45). São Paulo: Casa do Psicólogo.

Caballo, V. E. (2007). O treinamento de habilidades sociais. In: Caballo, V. E. (Org.). Manual de técnicas de terapia e modificação do comportamento. [Handbook of techniques of behavior therapy and mofication] (1th ed. pp.361-398). São Paulo: Santos.

Casali-Robalinho, I. G., Del Prette, Z. A. P., Del Prette, A. (2015). Habilidades Sociais como Preditoras de Problemas de Comportamento em Escolares. Psicologia: Teoria e Pesquisa, 31 (3), 321-330.

Cia, F., Pamplin, R. C. O., Del Prette, Z. A. P. (2006) Comunicação e participação paisfilhos: correlação com habilidades sociais e problemas de comportamento dos filhos. [Communication and parent- chilldren participation: a correlation with social skills and behavior problems of the children]. Paidéia. 16 (35), 385-406.

Dellagiustina, A.P.G., Broering, C.V. (2017). Tenha Modos. Foz do Iguaçu: Terapia Criativa.

Del Prette, Z. A. P., Del Prette, A. (2001) Habilidades sociais e educação: Pesquisa e atuação em psicologia escolar/educacional. In: Del Prette, Z. A. P. (Org) Psicologia escolar saúde e qualidade de vida. [School and educacional psychology: health and quality of life] (1th ed. pp.113-141). Campinas: Alínea.

Del Prette, Z. A. P., Del Prette, A. (2002) Avaliação de habilidades sociais de crianças com um inventario multimídia: indicadores sociométricos associados a frequência versus dificuldade. [Evaluation of children's social skills inventory: psichometric indexes of frequency versus difficulty] Psicologia em Estudo, Vol.7, 61-73.

Del Prette, Z. A. P., Del Prette, A. (2008) Psicologia das habilidades sociais na infância: teoria e prática. [Psychology of children social skills: theory and practice] Petrópolis: Vozes.

Del Prette, Z. A. P., Del Prette, A. (2012) Sistema Multimídia de Habilidades Sociais de Crianças: (SMHSC-manual). São Paulo: Casa do Psicólogo.

Del Prette, Z. A. P., Del Prette, A. (2009) Avaliação de habilidades sociais: Bases conceituais, instrumentos e procedimentos. A. Del Prette., Z. A. P. Del Prette (Orgs.), Psicologia das habilidades sociais: Diversidade teórica e suas implicações [Psychology of social skills: theoretical diversity and its implications] (pp.197-229). Petrópolis: Vozes.

Del Prette, A., Del Prette, Z. A. (2017). Psicologia das habilidades sociais na infância: teoria e prática. Petrópolis: Vozes.

Del Prette, Z. A., Del Prette, A. (2018). Competência social e habilidades sociais: manual teórico-prático. Petrópolis: Vozes.

LessaHorta, R., Schäfer, J.L., Coelho, L.R.M., Rodrigues, V.S., Oliveira, M.S., Teixeira, V. A. (2016). Condições associadas a prejuízo de desempenho em habilidades sociais em uma amostra de conveniência de usuários de crack. Cadernos de Saúde Pública, 32 (4). 
Linberger, J. (2017). Mulheres em tratamento pelo uso do crack: Habilidades socials e características clínicas (Dissertação de mestrado, Universidade do Vale do Rio dos Sinos, São Leopoldo, RS, Brasil).

Limberger, J., Andretta, I. (2017). Desenvolvimento das habilidades sociais na vida de mulheres usuárias de crack: estudo de casos múltiplos. Temas em Psicologia, 25(4), 1709-1724.

Longhini, L. Z., Rios, B.F., Peron, S., Neufeld, C.B. (2017). Caracterização das habilidades sociais de adolescentes em contexto escolar. Revista Brasileira de Terapias Cognitivas, 13(2), 131-137.

Murta, S. G., Goias, E. S. (2005) Aplicações do treinamento de habilidades sociais: análise da produção nacional. [Applications of training in social skills: analysis of social production] Psicologia: Reflexão e Crítica, 18 (2), 283-291.

Santos, E. B., Wachelke, J. (2019). Relações entre habilidades sociais de pais e comportamento dos filhos: uma revisão da literatura. Pesquisas e Práticas Psicossociais, 14(1), 1-15.

Siegel, S. (2015) Estatística não paramétrica para as ciências do comportamento. [non parametric Statistics for the Behaviorial Sciences] (1th ed. 350p.) São Paulo: McGrawHill Interamericana do Brasil, MGHB.

Silva, T.A., Cavalcante, L.I.C. (2017). Habilidades sociais infantis: comparações por gênero, idade e características escolares. Estudos e Pesquisas em Psicologia, 17 (2), 616-634.

Statsoft, Inc. (2011). STATISTICA (data analysis software system), version 10. www.statsoft.com. 